\title{
Protein Phosphorylation Site Prediction via Feature Discovery Support Vector Machine
}

\author{
Yi Shi*1, Bo Yuan ${ }^{2}$, Guohui Lin ${ }^{1}$, Dale Schuurmans ${ }^{1}$ \\ ${ }^{1}$ Department of Computing Science \\ University of Alberta. Edmonton, Alberta, Canada \\ \{ys3, guohui,daes\}@ualberta.ca \\ ${ }^{2}$ Department of Computer Science and Engineering \\ Shanghai Jiaotong University. Shanghai, China \\ yuanbo@cs.sjtu.edu.cn
}

\section{Abstract-Protein phosphorylation/dephosphorylation is}

the central mechanism of post-translational modification which regulates cellular responses and phenotypes. Due to the efficiency and resource constraints of the in vivo methods for identifying phosphorylation sites, there is a strong motivation to computationally predict potential phosphorylation sites. In this work, we propose to use a unique set of features to represent the peptides surrounding the amino acid sites of interest and use feature selection support vector machine to predict whether the serine/threonine sites are potentially phosphorylable, as well as selecting important features that may lead to phosphorylation. Experimental results indicate that the new features and the prediction method can more effectively predict protein phosphorylation sites than the existing state of the art methods. The features selected by the our prediction model provides biological insights to the in vivo phosphorylation.

Index Terms-Protein Phosphorylation, Support Vector Machine, Sparse Learning, Feature Selection, PositionSpecific Scoring Matrix.

\section{INTRODUCTION}

Protein phosphorylation happens in the posttranslational stage of prokaryotes and eukaryotes in which a protein kinase modifies a protein by adding a covalently bound phosphate group to a residue (usually serine, threonine or tyrosine). The protein regulation by phosphorylation, also known as phosphoregulation, is one of the most common regulations of protein function. A protein switches between a phosphorylated and an unphosphorylated form in almost all cases of phosphoregulation, and if one of these two is an active form, the other one is inactive. The phosphoregulation, which is essentially the transportation of energy groups, plays a central role in the regulation of almost all cellular behaviors [29], such as apoptosis [36], regulation of transcription [30], DNA repair [34], metabolism [2], cellular differentiation [15], environmental stress response [32], cellular mobility [24], and immune response [11]. Whether a protein has phosphorylized sites is mostly addressed, while which sites on a protein that are phosphorylated still remains challenging [21]. 
In vivo, researchers discovered novel phosphorylation sites mostly through low-throughput biological techniques. For example, many labs used the site-directed mutagenesis based technique to characterize specific phosphorylation events [19]. Such techniques are usually time-consuming and costly. More recently, the highthroughput mass spectrometry based technique has significantly accelerated the identification of novel sites [7]. Nonetheless, this technique also has limitations, and requires very expensive instruments and specialized expertise that are not available in typical laboratories [29].

Due to the limitations of both low-throughput and high-throughput in vivo techniques for phosphorylation site discovery, researchers are paying much attention on the in vitro approaches. These in vitro approaches usually only require a protein sequence as input, and output some quantified measurement that indicates how likely a serine, threonine or tyrosine $(\mathrm{S} / \mathrm{T} / \mathrm{Y})$ residue in that sequence is phosphorylated. Many computationally predicted phosphorylation sites have been experimentally validated in vivo [1], [4], [27].

Previous researches indicate that only when the amino acids surrounding a given $\mathrm{S} / \mathrm{T} / \mathrm{Y}$ residue fit certain patterns, the residue's phosphorylation can be catalyzed by a protein kinase [3]. However, sequence motifs that describe these patterns are neither sensitive nor specific (i.e., many patterns may occur at random) [1], [26]. Therefore, advanced machine learning methods that can identify more complex and subtle patterns are required. Many machine learning methods have been proposed which use position-specific scoring matrices (PSSMs), decision trees, genetic algorithms, artificial neural networks (ANNs), and support vector machines (SVMs). As the simplest technique, PSSM is a matrix in which rows represent amino acids and columns represent positions in a multiple sequence alignment; PSSM gives a weighted match to any given substring of fixed length. More complex PSSM variations are also developed in practice [12], [14]. However, PSSMs are not able to detect combinations of multiple amino acids patterns which may be important in practice [1]. The two most popular machine learning techniques, ANNs and SVMs, have proven to capture more complex patterns [1], thus are more widely adopted in phosphorylation site prediction, with the tradeoff of increasing computational complexity. In ANN classifiers, the classification function are usually implicit due to multiple neural layers and nonlinear weight functions [1], [9], [21]. In most SVM classifiers for protein phosphorylation site prediction, due to the adopted input usually being sparse coding of the peptides surrounding amino acid sites of interest, it is usually not clear which features essentially lead to the phosphorylation [5], [6], [10], [31], [33]. Some other methods employ the secondary structure [8] or 3D structural information [10], [16], [22], [25], [28].

After reviewing most machine learning methods in protein phosphorylation prediction, Trost et al. raise an important question: do they model the actual biological mechanisms underlying protein kinase recognition, or do they merely recognize patterns [29]? Trost et al. argue that the latter is more or less the case and propose that 
while pattern recognition has resulted in much success, it is plausible that more closely modeling the underlying biology of substrate recognition will result in the greater gains in predictive performance.

In this paper, we use 26 explicit sequence-based features for the phosphorylation site prediction. Meanwhile, to select important features that may provide insights to the phosphorylation mechanism, we use the $L_{1}$ norm support vector machine classifier. The rest of this paper is organized as follows: In Section II the detailed features and the classification models will be introduced; in Section III, we will introduce the dataset we used, the other methods we compare our method to, the evaluation criteria, and the experimental results; Section IV concludes the paper after discussion.

\section{MethodS}

\section{A. Features}

Rather than using sparse coded peptides as the input for a classifier, we use 26 explicit features for the peptides surrounding the amino acid of interest. The first 20 features are the 20 amino acid composition of the peptide, which is the occurrence of each amino acid divided by the peptide length. Although we lose some ordering information by treating each amino acid composition separately, considering the peptides we use are very short (e.g., 9-15 in length) and that the PSSM score will be used later, the information loss is not significant. Then we use the average hydrophobicity score and the average relative surface accessibility (RSA) score of the given peptide, that is, we sum up each individual amino acid's hydrophobicity (or RSA) score and divide it by the peptide length. The hydrophobicity scores and RSA scores are predicted by NetSurfP [23] using the whole protein sequence as inputs. The next 3 features are the percentage of the 3 predicted secondary structure composition, i.e., alpha helix, beta sheet, and random coil as they are believed to be important features in previous study [8]. Similar to the previous two scores, these three scores are also calculated based on NetSurfP's secondary structure predictions. The last feature we use is the position-specific scoring matrix (PSSM) score of a given peptide with respect to the positive training data [12], [14]. To calculate the PSSM score, we first construct the PSSM matrix based on the positive training data so that each row represents a symbol of the 20 amino acid alphabet and each column represents a position in the peptide. Each element of the matrix is the frequency of certain amino acid appearing in certain position. For a target peptide, its PSSM score is the sum of its amino acid in corresponding positions in the matrix. Note that the PSSM considers amino acid ordering information explicitly.

\section{B. $L_{1}$ norm SVM}

We use both $L_{2}$ norm SVM and $L_{1}$ norm support vector machines for predicting/classifying a given amino acid site's phosphorylation. The advantage of using $L_{2}$ norm SVM is that the original feature space can be mapped to higher dimensional space through kernelization, while the advantage of using $L_{1}$ norm SVM is that it can perform feature selection as well as classification. 
The $L_{1}$ norm SVM is thus what we mainly recommend to use for this work since we aim to not only do the phosphorylation site prediction but also aim to discover important features that are biologically relevant to the protein phosphorylation.

The primal form of $L_{1}$-norm SVM is:

$$
\begin{array}{cl} 
& \min _{\mathbf{w}, \mathbf{b}, \xi} \beta\|\mathbf{w}\|_{1}+\mathbf{1}^{T} \xi \\
\text { s.t. : } \quad & \xi \geq \mathbf{1}-\triangle(\mathbf{y})(X \mathbf{w}-\mathbf{1} b), \\
& \xi \geq \mathbf{0} .
\end{array}
$$

where $\triangle(\mathbf{y})$ denotes putting the vector $\mathbf{y}$ on the main diagonal of a square matrix. $X \in \mathbb{R}^{n \times p}, \mathbf{y} \in \mathbb{R}^{n}, n$ is the number of peptides, and $p$ is the number of features. Since

$$
\begin{array}{r}
\|\mathbf{w}\|_{1}=\min _{\gamma \geq 0} \frac{1}{2} \sum_{j}\left(\frac{w_{j}^{2}}{\gamma_{j}}+\gamma_{j}\right) \\
=\min _{\gamma \geq 0} \frac{1}{2}\left(\mathbf{w}^{T} G^{-1} \mathbf{w}+\gamma^{T} \mathbf{1}\right)
\end{array}
$$

[20], where $G=\triangle(\gamma)$, (1) becomes

$$
\begin{gathered}
\min _{\mathbf{w}, \mathbf{b}, \xi, \gamma} \frac{\beta}{2}\left(\mathbf{w}^{T} G^{-1} \mathbf{w}+\gamma^{T} \mathbf{1}\right)+\mathbf{1}^{T} \xi \\
\text { s.t. : } \quad \xi \geq \mathbf{1}-\triangle(\mathbf{y})(X \mathbf{w}-\mathbf{1} b), \\
\xi \geq \mathbf{0}, \gamma \geq \mathbf{0} .
\end{gathered}
$$

By applying Lagrangian multipliers and setting the partial derivative equal to $0,(2)$ becomes

$$
\begin{gathered}
\min _{\gamma} \max _{\lambda} \lambda^{T} \mathbf{1}-\frac{1}{2 \beta} \lambda^{T} \triangle(\mathbf{y}) X G X^{T} \triangle(\mathbf{y}) \lambda+\frac{\beta}{2} \gamma^{T} \mathbf{1} \\
\text { s.t. : } \quad \mathbf{0} \leq \lambda \leq \mathbf{1}, \\
\lambda^{T} \mathbf{y}=0, \\
\gamma \geq \mathbf{0 .}
\end{gathered}
$$

points and negative data points since machine learning methods work poorly on unbalanced datasets. Figure 1 demonstrates the frequency logos of the 20 amino acid composition in overall positive dataset (including both Serine and Threonine) and Serine-, Threonine-only positive dataset, while Figure 2 demonstrates logos of the negative datasets, respectively.

\section{B. Comparison Methods and Evaluation}

To compare our methods to existing methods, we (3) choose two widely recognized and representative meth- 


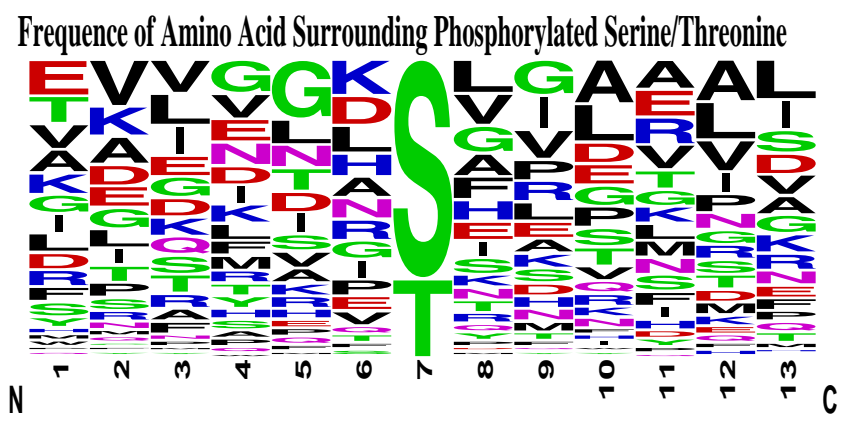

Frequence of Amino Acid Surrounding Phosphorylated Serine

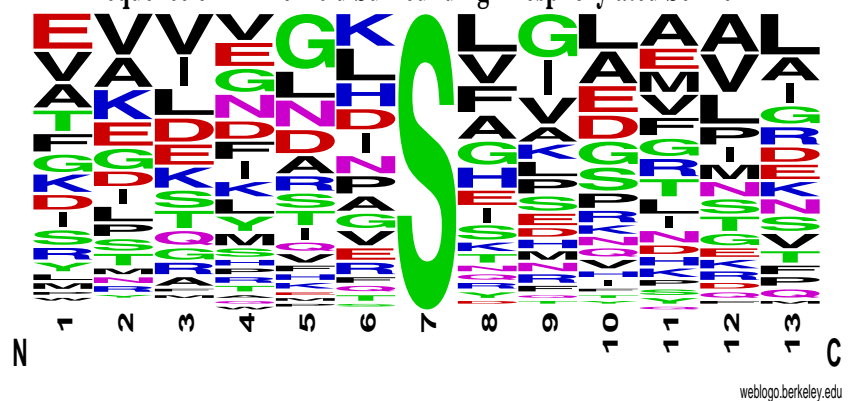

Frequence of Amino Acid Surrounding Phosphorylated Threonine
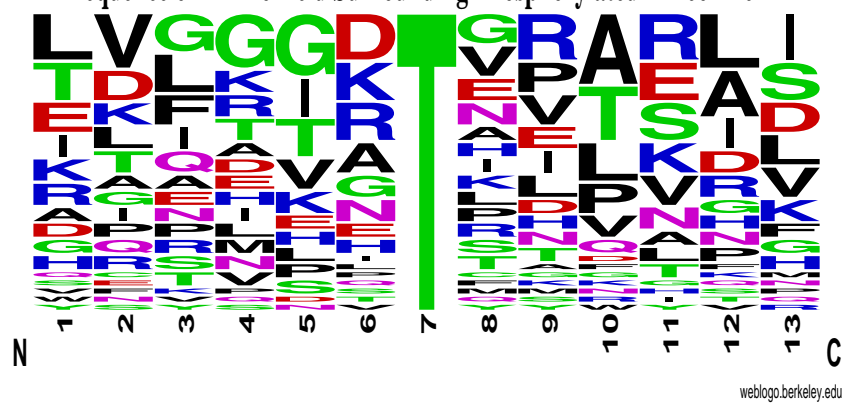

Fig. 1. The frequency $\log$ of the 20 amino acid composition in overall positive dataset (including both Serine and Threonine) and Serine-, Threonine-only positive dataset.

ods NetPhosBac [21] and NetPhos. NetPhosBac is designed specifically for predicting phosphorylation in bacteria data, while NetPhos is designed for Eukaryotic data. All the methods are performed under the 4-fold cross validation scheme described in [21] and are repeated 100 times. We also did the experiments on leave-oneout (LOO) cross validation, but since NetPhosBac and NetPhos were originally evaluated in 4-fold cross validation and the LOO results are not available, we only compare $L_{1}$ norm SVM and $L_{2}$ norm SVM (both linear
Frequence of Amino Acid Surrounding non-Phosphorylated Serine/Threonine

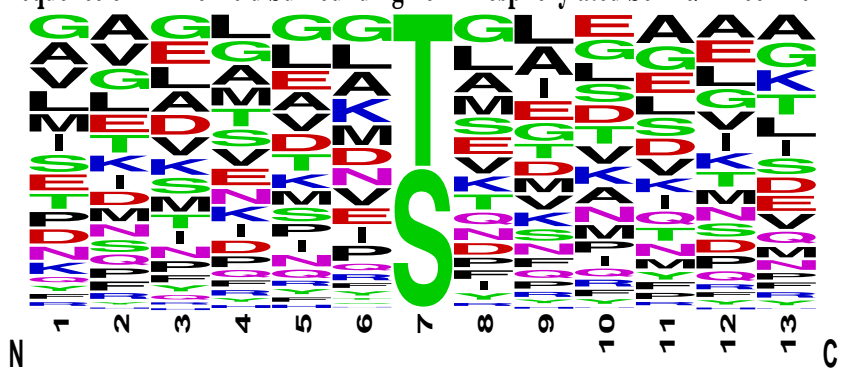

Frequence of Amino Acid Surrounding non-Phosphorylated Serine

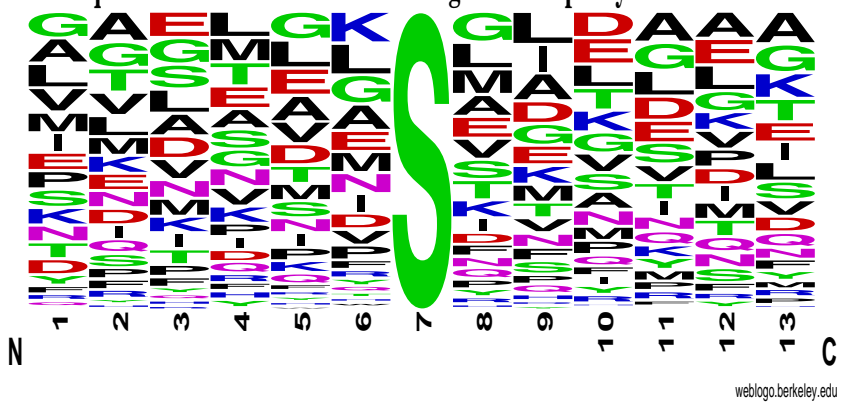

Frequence of Amino Acid Surrounding non-Phosphorylated Threonine

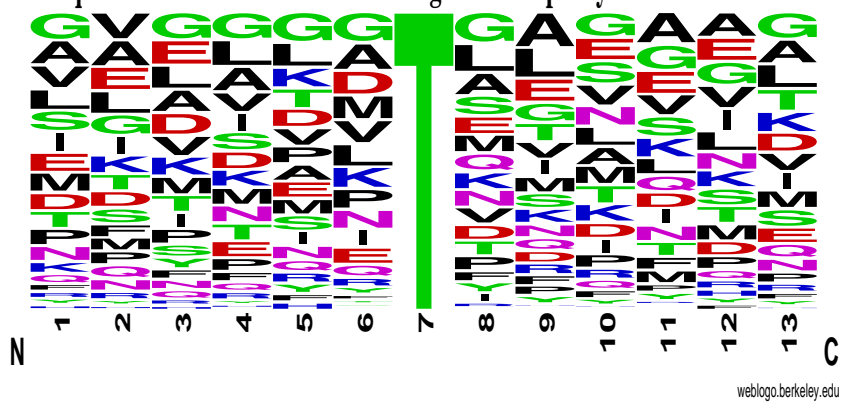

Fig. 2. The frequency logo of the 20 amino acid composition in overall negative dataset (including both Serine and Threonine) and Serine-, Threonine-only negative dataset.

kernel and RBF kernel) under the LOO cross validation setup.

We use various criteria to evaluate the effectiveness of different methods, including area under ROC curve (AROC), area under precision-recall curve (AUPR), Fmeasure, precision, and recall. The AROC and AUPR are appropriate performance measures for binary classification because it is not necessary to choose an arbitrary threshold for defining if a score signifies a positive or negative prediction. The precision and recall scores of 
each method are obtained at the cutoff threshold where the F-measure score is maximized.

\section{Results}

Tables I and II demonstrate the evaluation scores AROC, AUPR, FMeasure, Precision, and Recall, of the $L_{1}$ norm SVM, linear kernel $L_{2}$ norm SVM, RBF kernel $L_{2}$ norm SVM, NetPhosBac, and NetPhos prediction methods, under 4-fold and LOO cross validation setup respectively. The corresponding ROC curve plot and precision-recall curve plot are shown in Figures 3, 4, 5, and 6. The AROC, AUPR, and FMeasure scores indicate that the $L_{1}$ norm SVM is the most effective method for classification based on the 26 features we use, followed by linear kernel $L_{2}$ norm SVM and RBF kernel $L_{2}$ norm SVM. The NetPhosBac method performed less effective than the three SVM based approaches. Usually, the RBF kernel $L_{2} \mathrm{SVM}$ is believed to have better performance than linear kernel $L_{2}$ norm SVM, especially on datasets that have a small number of features, but it is not the case on this dataset.

Table III lists the values of the feature selection vector $w$ obtained from training the $L_{1}$ norm SVM on the whole dataset (140 positive data points and 841 negative data points). In principle, the greater the absolute value of a feature's $w$ value is, the more it is connected to the phosphorylation in vivo. For example, by investigating both Figures 1 and 2 and Table III, we can see that Arginine and Histidine have the largest $w$ values and indeed their frequencies are very different between their positive logo profiles and negative logo profiles. These

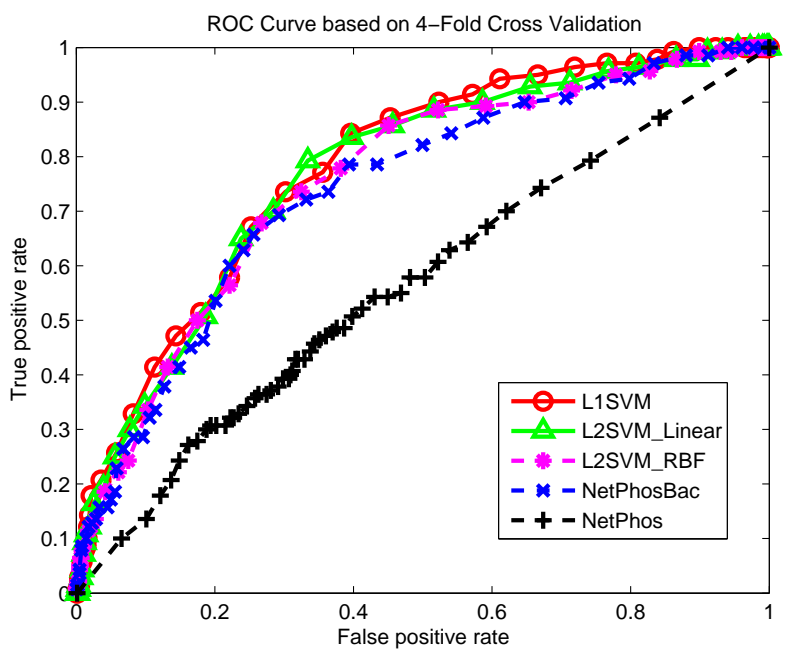

Fig. 3. The ROC curves of the prediction results of the $L_{1}$ norm SVM, linear kernel $L_{2}$ norm SVM, RBF kernel $L_{2}$ norm SVM, NetPhosBac, and NetPhos methods based on 4-fold cross validation setup.

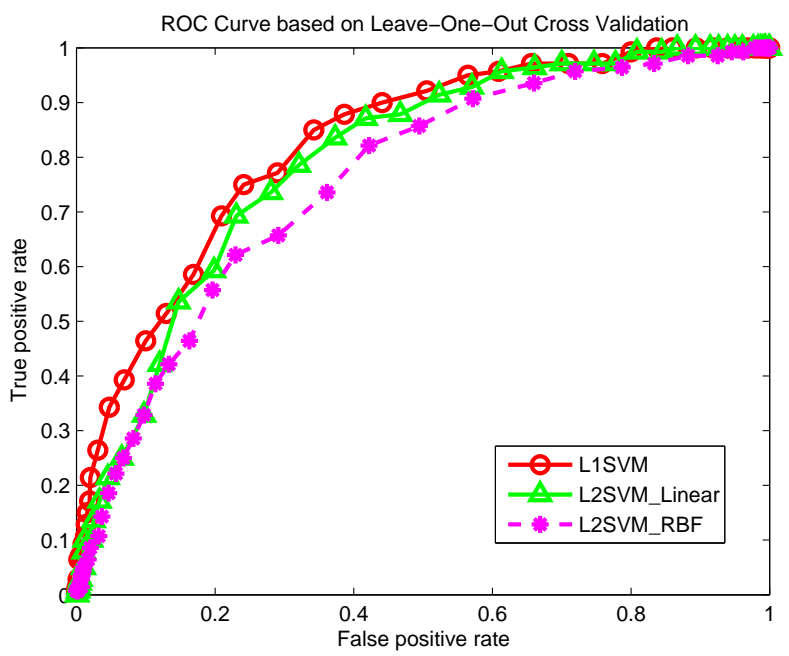

Fig. 4. The ROC curves of the prediction results of the $L_{1}$ norm SVM, linear kernel $L_{2}$ norm SVM, and RBF kernel $L_{2}$ norm SVM based on leave-one-out cross validation setup.

discoveries are consistent with the biochemistry principle as Arginine and Histidine both contain chemical group $\mathrm{NH}+$, which is an essential component in a phosphorylation process, because $S_{N} 2$ reaction in phosphorylating an -OH group requires the help of electron-neutralizing group, i.e., NH+. Methionine has the largest negative 


\begin{tabular}{|c||c|c|c|c|c|}
\hline Methods & AROC & AUPR & FMeasure & Precision & Recall \\
\hline L1SVM & $\mathbf{0 . 7 8 3 3}$ & $\mathbf{0 . 3 5 9 8}$ & $\mathbf{0 . 4 2 5 9}$ & $\mathbf{0 . 3 1 5 1}$ & 0.6571 \\
\hline L2SVM_Linear & 0.7708 & 0.3426 & 0.4152 & 0.3019 & $\mathbf{0 . 6 6 4 3}$ \\
\hline L2SVM_RBF & 0.7566 & 0.3401 & 0.4092 & 0.3017 & 0.6357 \\
\hline NetPhosBac & 0.7416 & 0.3340 & 0.4116 & 0.2997 & 0.6571 \\
\hline NetPhos & 0.5606 & 0.2369 & 0.2610 & 0.1785 & 0.4857 \\
\hline
\end{tabular}

TABLE I

The eVAluation scores AROC, AUPR, FMeAsure, Precision, AND ReCAll, of the $L 1$ NORM SVM, LiNEAR KERNEL $L 2$ NORM SVM, RBF KERNEL L2 NORM SVM, NETPHOSBAC, AND NETPHOS PREDICTION METHODS, UNDER 4-FOLD CROSS VALIDATION SETUP.

\begin{tabular}{|c||c|c|c|c|c|}
\hline Methods & AROC & AUPR & FMeasure & Precision & Recall \\
\hline L1SVM & $\mathbf{0 . 8 2 4 2}$ & $\mathbf{0 . 4 3 5 3}$ & $\mathbf{0 . 4 6 5 1}$ & $\mathbf{0 . 3 4 4 8}$ & 0.7143 \\
\hline L2SVM_Linear & 0.7965 & 0.3606 & 0.4289 & 0.2981 & $\mathbf{0 . 7 6 4 3}$ \\
\hline L2SVM_RBF & 0.7571 & 0.3174 & 0.4027 & 0.2932 & 0.6429 \\
\hline
\end{tabular}

TABLE II

The eVAluation scores AROC, AUPR, FMeAsure, Precision, AND ReCAll, of the $L 1$ NORM SVM, LiNEAR KeRnel L2 NORM SVM, RBF KERNEL L2 NORM SVM, NETPHOSBAC, AND NETPHOS PREDICTION METHODS, UNDER LEAVE-ONE-OUT CROSS

VALIDATION SETUP.

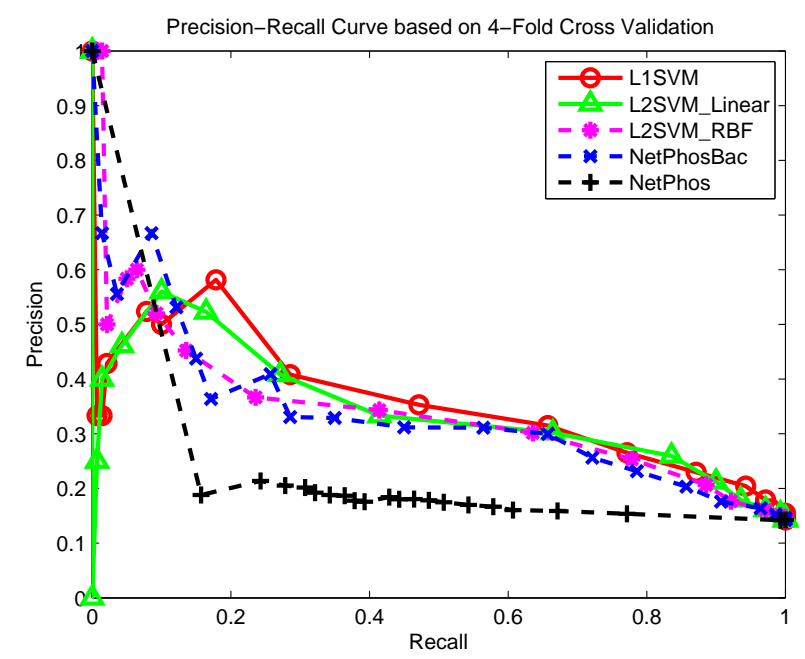

Fig. 5. The precision-recall curves of the prediction results of the $L_{1}$ norm SVM, linear kernel $L_{2}$ norm SVM, RBF kernel $L_{2}$ norm SVM, NetPhosBac, and NetPhos methods based on 4-fold cross validation setup.

value and indeed it appears much more frequently in negative data points than in positive data points. Also, according to the $w$ values, hydrophobicity score isn't a very significant feature; relative surface accessibility

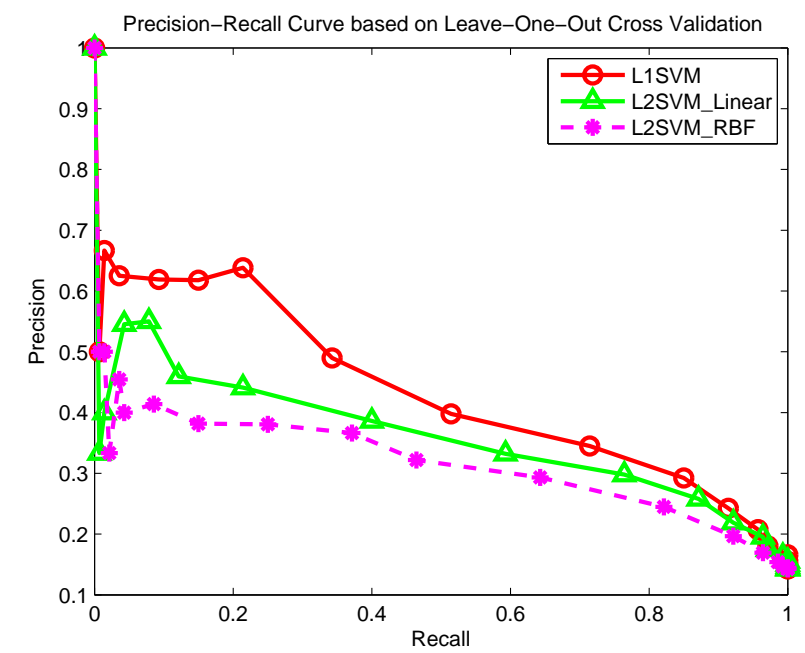

Fig. 6. The precision-recall curves of the prediction results of the $L_{1}$ norm SVM, linear kernel $L_{2}$ norm SVM, and RBF kernel $L_{2}$ norm SVM methods based on leave-one-out cross validation setup.

(RSA) score is fairly important; Alpha helix and random coil appear more in negative data points than in positive data points; the PSSM score is an important feature which is in accord with common sense. Table III also lists the correlation coefficient between each individual 
feature and the training label. We can see that the correlation coefficient scores associated with the features are strongly connected to the trained $w$ values. We calculated the correlation coefficient score between the $w$ vector and coef. vector, which is 0.75 and re-affirms their connections. Based on these, we may argue that our $L_{1}$ norm SVM can not only predict the protein phosphorylation sites more accurately, but also select the important features that are connected to the underlining biological reasons that cause protein phosphorylation.

\section{Discussion AND CONClusion}

In this work, we propose to use a unique set of features to represent the peptides surrounding the amino acid sites that may be potentially phosphorylated. We use $L_{1}$ norm feature selection support vector machine to predict whether the sites are phosphorylable, as well as selecting important features that may cause the phosphorylation. The feature selection functions in our prediction model were never investigated before to the best of our knowledge, making our method a novel approach in protein phosphorylation prediction area. Experimental results indicate that the features and the prediction method can more effectively predict protein phosphorylation than existing state of the art methods. The features selected by the our prediction model provide insights into the biochemical cause of protein phosphorylation in vivo.

\section{REFERENCES}

[1] N. Blom, S. Gammeltoft, and S. Brunak1. Sequence and structure-based prediction of eukaryotic protein phosphorylation sites. Journal of Molecular Biology, 294:1356-1362, 1999.
[2] Y. H. Bu, Y. L. He, H. D. Zhou, W. Liu, D. Peng, A. G. Tang, L. L. Tang, H. Xie, Q. X. Huang, X. H. Luo, and E. Y. Liao. Insulin receptor substrate 1 regulates the cellular differentiation and the matrix metallopeptidase expression of preosteoblastic cells. J. Endocrinol., 206:271-277, 2010.

[3] S. H. Diks, K. Parikh, M. V. D. Sijde, J. Joore, T. Ritsema, and M. P. Peppelenbosch. Evidence for a minimal eukaryotic phosphoproteome. PLoS One, 2:e777, 2007.

[4] H. C. Fan, X. Zhang, and P. A. McNaughton. Activation of the TRPV4 ion channel is enhanced by phosphorylation. J. Biol. Chem., 284:27884-27891, 2009.

[5] F. Gnad, S. Ren, J. Cox, J. V. Olsen, B. Macek, M. Oroshi, and M. Mann. PHOSIDA (phosphorylation site database): management, structural and evolutionary investigation, and prediction of phosphosites. Genome Biol., 8:R250, 2007.

[6] J. L. Heazlewood, P. Durek, J. Hummel, J. Selbig, W. Weckwerth, D. Walther, and W. X. Schulze. PhosPhAt: a database of phosphorylation sites in arabidopsis thaliana and a plantspecific phosphorylation site predictor. Nucleic Acids Res., 36:D1015-D1021, 2008.

[7] E. L. Huttlin, M. P. Jedrychowski, J. E. Elias, T. Goswami, R. Rad, S. A. Beausoleil, J. Villen, W. Haas, M. E. Sowa, and S. P. Gygi. A tissue-specific atlas of mouse protein phosphorylation and expression. Cell, 143:1174-1189, 2010.

[8] L. M. Iakoucheva, P. Radivojac, C. J. Brown, T. R. O'connor, J. G. Sikes, Z. Obradovic, and A. K. Dunker. The importance of intrinsic disorder for protein phosphorylation. Nucleic Acids Res., 32:1037-1049, 2004.

[9] C. R. Ingrell, M. L. Miller, O. N. Jensen, and N. Blom. NetPhos Yeast: prediction of protein phosphorylation sites in yeast. Bioinformatics, 23:895-897, 2007.

[10] J. H. Kim, J. Lee, B. Oh, K. Kimm, and I. Koh. Prediction of phosphorylation sites using SVMs. Bioinformatics, 20:31793184, 2004.

[11] S. H. Kim and C. E. Lee. Counter-regulation mechanism of IL4 and IFN-signal transduction through cytosolic retention of the pY-STAT6:pY-STAT2:p48 complex. Eur. J. Immunol., 41:461472, 2011.

[12] M. Koenig and N. Grabe. Highly specific prediction of phos- 


\begin{tabular}{|c|c|c||c|c|c|}
\hline Feature & $\mathrm{W}$ & Coef. & Feature & $\mathrm{W}$ & Coef. \\
\hline \hline Alanine & -0.783 & -0.030 & Phenylalanine & 0.00 & 0.006 \\
\hline Arginine & 2.444 & 0.194 & Proline & 0.00 & -0.020 \\
\hline Asparagine & -0.087 & -0.030 & Serine & 0.00 & 0.080 \\
\hline Aspartic & -0.057 & 0.001 & Threonine & -0.011 & -0.111 \\
\hline Cysteine & 0.00 & 0.094 & Tryptophan & 0.00 & 0.038 \\
\hline Glutamic & -0.482 & -0.031 & Tyrosine & -0.537 & -0.07 \\
\hline Glutamine & -0.232 & -0.063 & Valine & 0.034 & 0.088 \\
\hline Glycine & -0.551 & 0.00 & Hydrophob. & 0.049 & 0.005 \\
\hline Histidine & 2.453 & 0.129 & RSA & 0.179 & -0.009 \\
\hline Isoleucine & 0.00 & 0.055 & Alpha Helix & -0.610 & 0.055 \\
\hline Leucine & -0.503 & 0.031 & Beta Sheet & 0.00 & -0.002 \\
\hline Lysine & 0.061 & 0.003 & Coil & -1.670 & -0.070 \\
\hline Methionine & -2.965 & -0.188 & PSSM Score & 0.738 & 0.237 \\
\hline
\end{tabular}

TABLE III

THE VALUES OF THE FEATURE SELECTION VECTOR $W$, AND THE CORRELATION COEFFICIENT VALUES BETWEEN EACH FEATURE VECTOR AND THE TRAINING LABEL VECTOR.

phorylation sites in proteins. Bioinformatics, 20:3620-3627, 2004.

[13] G. Lanckriet, M. Cristianini, P. Bartlett, and et al. Learning the kernel matrix with semi-definite programming. J. Mach. Learn. Res., 5:27-72, 2004.

[14] T. Li, F. Li, and X. Zhang. Prediction of kinase-specific phosphorylation sites with sequence features by a log-odds ratio approach. Proteins, 70:404-414, 2008.

[15] I. Lian, J. Kim, H. Okazawa1, J. Zhao, B. Zhao, J. Yu, A. Chinnaiyan, M. A. Israel, L. S. B. Goldstein, R. Abujarour, S. Ding, and K. L. Guan. The role of YAP transcription coactivator in regulating stem cell self-renewal and differentiation. Genes Dev., 24:1106-1118, 2010.

[16] R. Linding, L. J. Jensen, G. J. Ostheimer, M. A. van Vugt, C. Jorgensen, I. M. Miron, F. Diella, K. Colwill, L. Taylor, K. Elder, P. Metalnikov, V. Nguyen, A. Pasculescu, J. Jin, J. G. Park, L. D. Samson, J. R. Woodgett, R. B. Russell, P. Bork, M. B. Yaffe, and T. Pawson. Systematic discovery of in vivo phosphorylation networks. Cell, 129:1415-1426, 2007.

[17] B. Macek, F. Gnad, B. Soufi, C. Kumar, J. V. Olsen, I. Mijakovic, and M. Mann. Phosphoproteome analysis of e. coli reveals evolutionary conservation of bacterial Ser/Thr/Tyr phosphorylation. Mol. Cell. Proteomics, 7:299-307, 2008.
[18] B. Macek, I. Mijakovic, J. V. Olsen, F. Gnad, C. Kumar, P. R. Jensen, and M. Mann. The serine/threonine/tyrosine phosphoproteome of the model bacterium bacillus subtilis. Mol. Cell. Proteomics, 6:697-707, 2007.

[19] R. Meier, D. R. Alessi, P. Cron, M. Andjelkovic, and B. A. Hemmings. Mitogenic activation, phosphorylation, and nuclear translocation of protein kinase beta. J. Biol. Chem., 272:3049130497, 1997.

[20] C. A. Micchelli and M. Pontil. Learning the kernel function via regularization. J. Mach. Learn. Res., 6:1099-1125, 2006.

[21] M. L. Miller, B. Soufi, C. Jers, N. Blom, B. Macek, and I. Mijakovic. Netphosbac - a predictor for ser/thr phosphorylation sites in bacterial proteins. Proteomics, 9:116-125, 2009.

[22] G. Neuberger, G. Schneider, and F. Eisenhaber. pkaPS: prediction of protein kinase A phosphorylation sites with the simplified kinase-substrate binding model. Biol. Direct., 2:1, 2007.

[23] B. Petersen, T. N. Petersen, P. Andersen, M. Nielsen, and C. Lundegaard. A generic method for assignment of reliability scores applied to solvent accessibility predictions. BMC Struct Biol, 9:51, 2009.

[24] M. Ressurrecao, D. Rollinson, A. M. Emery, and A. J. Walker. A role for $\mathrm{p} 38 \mathrm{MAPK}$ in the regulation of ciliary motion in a 
eukaryote. BMC Cell Biol., 12:6, 2011.

[25] N. F. W. Saunders and B. Kobe. The Predikin webserver: improved prediction of protein kinase peptide specificity using structural information. Nucleic Acids Res., 36:W286-W290, 2008.

[26] C. J. Sigrist, L. Cerutti, N. Hulo, A. Gattiker, L. Falquet, M. Pagni, A. Bairoch, and P. Bucher. PROSITE: a documented database using patterns and profiles as motif descriptors. Bioinformatics, 18:265-274, 2002.

[27] S. A. Slaugenhaupt, A. Blumenfeld, S. P. Gill, M. Leyne, J. Mull, M. P. Cuajungco, C. B. Liebert, B. Chadwick, M. Idelson, L. Reznik, C. M. Robbins, . M. J. B. Izabela Makalowska, D. Krappmann, C. Scheidereit, C. Maayan, F. B. Axelrod, and J. F. Gusella. Tissue-specific expression of a splicing mutation in the IKBKAP gene causes familial dysautonomia. Am. J. Hum. Genet., 68:598-605, 2001.

[28] K. Swaminathan, R. Adamczak, A. Porollo, and J. Meller. Enhanced prediction of conformational flexibility and phosphorylation in proteins. Adv. Exp. Med. Biol., 680:307-319, 2010.

[29] B. Trost and A. Kusalik. Computational prediction of eukaryotic phosphorylation sites. Bioinformatics, 27:2927-2935, 2011.

[30] S. Uddin, F. Lekmine, A. Sassano, H. Rui, E. N. Fish, and L. C. Platanias. Role of Stat5 in type I interferon-signaling and transcriptional regulation. Biochem. Biophys. Res. Commun., 308:325-330, 2003.

[31] M. Wang, C. Li, W. Chen, and C. Wang. Prediction of PKspecific phosphorylation site based on information entropy. Sci. China C Life Sci., 51:12-20, 2008.

[32] Y. Y. Wang, S. M. Chen, and H. Li. Hydrogen peroxide stress stimulates phosphorylation of foxo1 in rat aortic endothelial cells. Acta Pharmacol. Sin., 31:160-164, 2010.

[33] Y. H. Wong, T. Y. Lee, H. K. Liang, C. M. Huang, T. Y. Wang, Y. H. Yang, C. H. Chu, H. D. Huang, M. T. Ko, and J. K. Hwang. KinasePhos 2.0: a web server for identifying protein kinasespecific phosphorylation sites based on sequences and coupling patterns. Nucleic Acids Res., 35:W588-W594, 2007.

[34] C. D. Wood, T. M. Thornton, G. Sabio, R. A. Davis, and M. Rincon. Nuclear localization of p38MAPK in response to DNA damage. Int. J. Biol. Sci., 5:428-437, 2009.
[35] S. M. Wurgler-Murphy, D. M. King, and P. J. Kennelly. The phosphorylation site database: A guide to the serine-, threonine-, and/or tyrosine-phosphorylated proteins in prokaryotic organisms. Proteomics, 4:1562-1570, 2004.

[36] J. Zhang and G. V. Johnson. Tau protein is hyperphosphorylated in a site-specific manner in apoptotic neuronal PC12 cells. $J$. Neurochem., 75:2346-2357, 2000. 